\title{
KNOW THE STAR, KNOW THE PLANET. V. CHARACTERIZATION OF THE STELLAR COMPANION TO THE EXOPLANET HOST STAR HD 177830
}

\author{
Lewis C. Roberts Jr. ${ }^{1}$, Rebecca Oppenheimer ${ }^{2}$, Justin R. CrepP ${ }^{3}$, Christoph Baranec ${ }^{4}$, Charles Beichman ${ }^{1,5,6}$, \\ Douglas Brenner ${ }^{2}$, Rick Burruss ${ }^{1}$, Eric Cady ${ }^{1}$, Statia Luszcz-CoOK ${ }^{2}$, Richard Dekany ${ }^{5}$, \\ Lynne Hillenbrand ${ }^{5}$, Sasha Hinkley ${ }^{7}$, David King ${ }^{8}$, Thomas G. Lockhart ${ }^{1}$, Ricky Nilsson ${ }^{2,9}$, IAN R. Parry ${ }^{8}$, \\ Laurent Pueyo ${ }^{10,11}$, Anand Sivaramakrishnan ${ }^{9}$, Rémi Soummer ${ }^{9}$, Emily L. Rice ${ }^{12}$, Aaron Veicht ${ }^{2}$, \\ Gautam Vasisht $^{1}$, Chengxing Zhai ${ }^{1}$, and Neil T. Zimmerman ${ }^{13}$ \\ ${ }^{1}$ Jet Propulsion Laboratory, California Institute of Technology, 4800 Oak Grove Drive, Pasadena CA 91109, USA; lewis.c.roberts@jpl.nasa.gov \\ ${ }^{2}$ American Museum of Natural History, Central Park West at 79th Street, New York, NY 10024, USA \\ ${ }^{3}$ Department of Physics, University of Notre Dame, 225 Nieuwland Science Hall, Notre Dame, IN 46556, USA \\ ${ }^{4}$ Institute for Astronomy, University of Hawai'i at Mānoa, Hilo, HI 96720-2700, USA \\ ${ }^{5}$ Division of Physics, Mathematics, and Astronomy, California Institute of Technology, Pasadena, CA 91125, USA \\ ${ }^{6}$ NASA Exoplanet Science Institute, 770 S. Wilson Avenue, Pasadena, CA 911225, USA \\ ${ }^{7}$ School of Physics, University of Exeter, Stocker Road, Exeter, EX4 4QL, UK \\ ${ }^{8}$ Institute of Astronomy, University of Cambridge, Madingley Road., Cambridge, CB3 OHA, UK \\ ${ }^{9}$ Department of Astronomy, Stockholm University, AlbaNova University Center, Roslagstullsbacken 21, SE-10691 Stockholm, Sweden \\ ${ }^{10}$ Space Telescope Science Institute, 3700 San Martin Drive, Baltimore, MD 21218, USA \\ ${ }^{12}$ Department of Engineering Science and Physics, College of Staten Island, City University of New York, Staten Island, NY 10314, USA \\ ${ }^{13}$ Princeton University, MAE, D207 Engineering Quad, Princeton, NJ 08544, USA \\ Received 2015 June 10; accepted 2015 July 28; published 2015 September 8
}

\begin{abstract}
HD 177830 is an evolved K0IV star with two known exoplanets. In addition to the planetary companions it has a late-type stellar companion discovered with adaptive optics imagery. We observed the binary star system with the PHARO near-IR camera and the Project 1640 coronagraph. Using the Project 1640 coronagraph and integral field spectrograph we extracted a spectrum of the stellar companion. This allowed us to determine that the spectral type of the stellar companion is a M4 $\pm 1 \mathrm{~V}$. We used both instruments to measure the astrometry of the binary system. Combining these data with published data, we determined that the binary star has a likely period of approximately 800 years with a semimajor axis of 100-200 AU. This implies that the stellar companion has had little or no impact on the dynamics of the exoplanets. The astrometry of the system should continue to be monitored, but due to the slow nature of the system, observations can be made once every 5-10 years.
\end{abstract}

Key words: binaries: visual - instrumentation: adaptive optics - stars: individual (HD 177830)

Supporting material: animation

\section{INTRODUCTION}

HD 177830 (HIP $93746=$ WDS 19053+2555) is an evolved K0IV star (Vogt et al. 2000) that is on the boundary between giants and subgiants (Ghezzi et al. 2010). It is a nearby star with a distance of $59.0 \pm 2.2 \mathrm{~s} \mathrm{pc}$ (van Leeuwen 2007). There have been several age determinations including an age of $3.8_{-1.6}^{+2.8} \mathrm{Gyr}$ (Valenti \& Fischer 2005), 4.03 Gyr (Saffe et al. 2005), 3.28 $8_{-0.24}^{+0.36} \mathrm{Gyr}$ (Takeda et al. 2007), and $3.46 \pm$ $0.29 \mathrm{Gyr}$ (Jofré et al. 2015) all of which are consistent. Baines et al. (2008a) measured the diameter of the star interferometrically with the CHARA Array as $2.99 \pm 0.39 R_{\odot}$ and estimated the $T_{\text {eff }}$ as $4804 \mathrm{~K}$. Using high resolution visible spectra, Mortier et al. (2013) measured the metallicity, $\log g$, $T_{\text {eff }}$, and $\xi_{t}$ and then used these parameters to estimate the mass using theoretical isochrones and a Bayesian estimation method (de Silva et al. 2006). This resulted in a mass of $1.17 \pm 0.10$ $M_{\odot}$, and $T_{\text {eff }}$ as $4752 \pm 79 \mathrm{~K}$. Jofré et al. (2015) carried out a similar analysis using different input spectra and modeling tools to derive the input parameters, but used the same software to compute masses. Their estimates of $1.37 \pm 0.04 M_{\odot}$ and $T_{\text {eff }}$ of $5058 \pm 35 \mathrm{~K}$ are slightly different. The system has been observed with multiple instruments on the Spitzer telescope and none of the observations have turned up any evidence of a debris disk (Beichman et al. 2005; Trilling et al. 2008; Bryden et al. 2009; Tanner et al. 2009; Dodson-Robinson et al. 2011).

By monitoring radial velocity (RV) variations, Vogt et al. (2000) discovered a planet orbiting HD 177830 with a period of 391.6 days, an eccentricity of 0.41 , and an $M \sin i$ of $1.22 M_{J}$, where $M$ is the mass and $i$ is the inclination. This planet was designated HD $177830 \mathrm{~b}$. It orbits the primary at a distance of $0.63-1.57 \mathrm{AU}$ and lies inside the habitable zone. The planetary orbit was updated by Butler et al. (2006) and again by Wright et al. (2007) who also showed the first signs of a second planet in the system. The star continued to be observed and Meschiari et al. (2011) published an update of the orbital parameters of HD $177830 \mathrm{~b}$ and announced the discovery of an inner planet in the system. This planet, HD 177830 c, has a period of $110.9 \pm 0.1$ days and eccentricity of $0.3 \pm 0.1$ and an $M \sin i$ of $0.15 \pm 0.02 M_{J}$. The updated parameters of HD $177830 \mathrm{~b}$ are a period of 407.31 days, an eccentricity of $0.009 \pm 0.004$, and an $M \sin i$ of $1.48 M_{J}$. Reffert \& Quirrenbach (2011) were able to use the lack of astrometric detection with Hipparcos satellite to place an upper limit on the mass of HD $177830 \mathrm{~b}$ of $225.2 M_{J}$.

Eggenberger et al. (2007) first reported the discovery of a stellar companion to HD 177830 with a separation of 1". 6 using adaptive optics (AO) on the Very Large Telescope. The stellar 
Table 1

Measured Astrometry and Photometry for HD 177830

\begin{tabular}{|c|c|c|c|c|}
\hline Epoch & $\theta\left(^{\circ}\right)$ & $\rho\left({ }^{\prime \prime}\right)$ & $\Delta M(\mathrm{Ks})$ & Instrument/Reference \\
\hline 2002.5474 & $84.1 \pm 1.0$ & $1.62 \pm 0.01$ & $\ldots$ & Roberts et al. (2011) \\
\hline 2004.4784 & $84.85 \pm 0.21$ & $1.645 \pm 0.008$ & $\ldots$ & Eggenberger et al. (2007) \\
\hline 2005.3494 & $84.60 \pm 0.39$ & $1.64 \pm 0.01$ & $\ldots$ & Eggenberger et al. (2007) \\
\hline 2012.3549 & $84.3 \pm 0.2$ & $1.67 \pm 0.01$ & $6.5 \pm 0.3$ & PHARO \\
\hline 2012.4475 & $86.0 \pm 0.1$ & $1.68 \pm 0.002$ & $\ldots$ & P1640 \\
\hline 2014.6748 & $85.3 \pm 0.2$ & $1.67 \pm 0.01$ & $6.1 \pm 0.3$ & PHARO \\
\hline
\end{tabular}

companion was designated HD $177830 \mathrm{~B}$, while the primary was designated HD $177830 \mathrm{~A}$. Based on near-IR colors and the differential magnitude of 6.6 at $1.6 \mu \mathrm{m}$ they estimated the spectral type of HD $177830 \mathrm{~B}$ to be M2V-M5V with a mass of $0.23 \pm 0.01 M_{\odot}$. Roberts et al. (2011) published an observation of the same companion that predates the discovery images of Eggenberger et al. (2007) using the visible-light AO system on the Advanced Electro-Optical System (AEOS) telescope. The astrometry from both papers is shown in Table 1.

There have been several prior high angular resolution observations of the HD 177830. Baines et al. (2008b) used the CHARA Array to search for stellar companions and did not detect the stellar companion because it was outside of the instrument's field of view of 0 ". 1 . Inside of their field of view, they were able to rule out any additional stellar companions earlier than K0V. Lu et al. (1987) carried out speckle interferometry observations with the Kitt Peak $4 \mathrm{~m}$ telescope, but the observations did not achieve a sufficient dynamic range to detect the companion.

The stellar companion's measured separation corresponds to a projected separation of $97 \mathrm{AU}$ and this separation has the potential to have an impact on the dynamical behavior and evolution of the exoplanets orbiting HD 177830 A. The population of exoplanets in binaries with semimajor axes smaller than $100 \mathrm{AU}$ is statistically different than those orbiting single stars (Zucker \& Mazeh 2002; Bonavita \& Desidera 2007), while for binaries with separations larger than $100 \mathrm{AU}$ the frequencies of exoplanets among single stars and components of wide binaries are indistinguishable (Raghavan et al. 2006; Bonavita \& Desidera 2007). With that in mind, we carried out observations with two instruments on the Palomar $5 \mathrm{~m}$ telescope in an effort to understand the orbital parameters of this exoplanet hosting binary star.

\section{OBSERVATIONS AND DATA ANALYSIS}

\subsection{Project 1640}

HD 177830 was observed with the Project 1640 (P1640) coronagraph (Hinkley et al. 2011) on 2012 June 12 UT. The P1640 coronagraph is integrated with an integral field spectrograph covering the $Y J H$ bands. Like PHARO, this instrument is mounted on the PALM-3000 AO system. We collected three data cubes, each with an integration time of 549.9 s. For each data cube the primary was placed behind the occulting disk. The data were reduced using the Project 1640 data reduction pipeline (Zimmerman et al. 2011). Since that paper was written, a few upgrades have been made to the pipeline that reduce lenslet-lenslet cross talk. The pipeline processing produces an image of the object in each of 32 wave bands resulting in a data cube.

While we collected several unocculted images of HD 177830, the dynamic range between the companion and primary makes it extremely difficult to detect the companion. Two of our occulted images used the astrometric grid spots (Sivaramakrishnan \& Oppenheimer 2006). We fit a Gaussian to each of these spots. Then we identified the location of the primary by fitting lines to the vertical and the horizontal spots. The primary is located where these two lines intersect. Figure 1 shows an image slice with the grid spots. The final astrometry is the average of the astrometry measured from 26 of the data slices from each of the two data cubes with grid spots. The frames in the water absorbtion bands with center wavelengths of $1145,1170,1395,1420,1445$, and $1795 \mathrm{~nm}$ had too low signal to fit a Gaussian to the grid spots. The astrometry is presented in Table 1, which lists the Besselian date of the observation, the position angle $(\theta)$ and separation $(\rho)$ of the system and the instrument used in the observation.

\subsection{PHARO}

We observed HD 177830 on 2012 May 9 UT and on 2014 May 14 UT with the Palomar Observatory Hale $5 \mathrm{~m}$ telescope using the PALM-3000 AO system and the PHARO near-IR camera. The PALM-3000 AO system is a natural guide star system using two deformable mirrors (DM) (Dekany et al. 2013). One DM corrects low-amplitude high spatial frequency aberrations, while the other corrects the higher-amplitude low spatial frequency aberrations. The system is optimized for high contrast observations and routinely produces Strehl ratios greater than $80 \%$ in the $K s$ band. The PHARO camera uses a $\mathrm{HgCdTe}$ HAWAII detector for observations between 1 and $2.5 \mu \mathrm{m}$ wavelengths (Hayward et al. 2001). The camera has multiple filters in two filter wheels and we used the $K s$ filter to collect 10 frames in 2012 and 50 frames in 2014. In 2012, only sky frames were collected for calibration purposes. Past experience with PHARO has shown that its flat fields and dark frames are very stable from run to run. We used calibration data from 2013 to calibrate the 2012 images. Calibration frames were collected on the same night as the 2014 data. The night of 2014 May 14 UT suffered from high winds resulting in poor seeing and lower image quality than is normal for P3K. After debiasing, flat fielding, bad pixel correction, and background subtraction, the frames were coadded. The resulting images are shown in Figure 2. The fitstars algorithm was used to measure the astrometry and photometry of the objects (ten Brummelaar et al. 1996, 2000). Photometric error bars were assigned using the technique described in Roberts et al. (2005). Astrometric error bars were set equal to the median error bar of 26 binaries measured with P3K and PHARO (Roberts et al. 2015). The resulting measurements are in Table 1 . It has the same format as the P1640 results, but we also list the differential magnitude between the two stars in the $K s$ filter. 


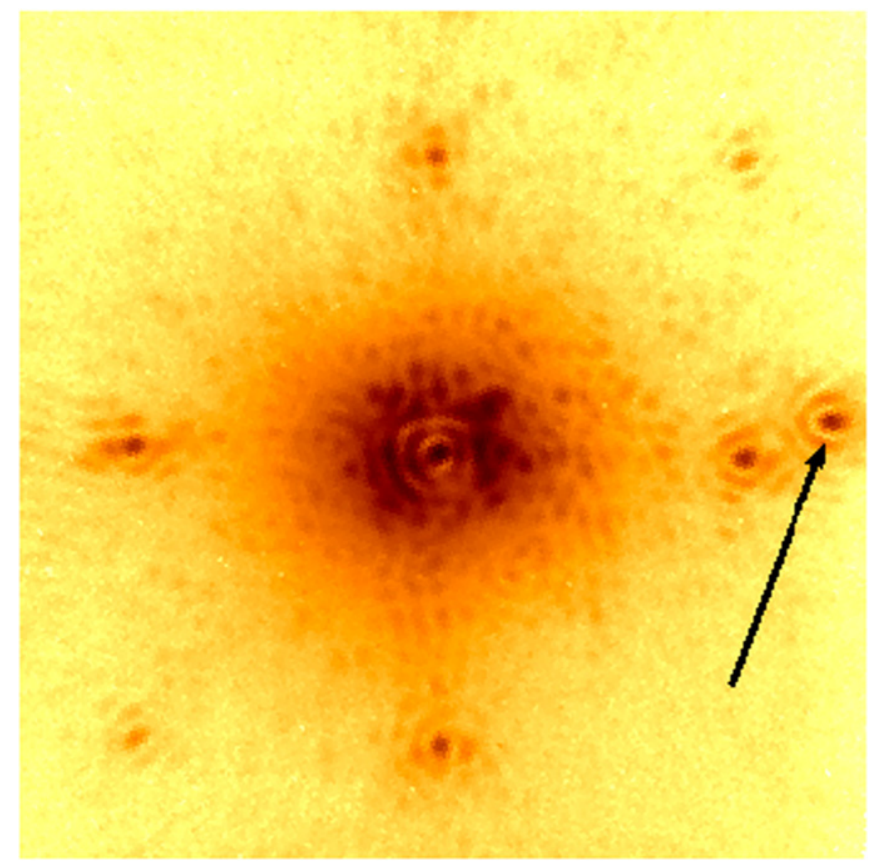

Figure 1. Slice of the occulted P1640 image cube at a wavelength of $1.495 \mu \mathrm{m}$. It has been rotated so that north is up and east is to the right. There are four astrometric grid spots in the image, see the text for a discussion of their use. An arrow points toward the companion. The field of view is 4 ". 0 .

(An animation of this figure is available.)

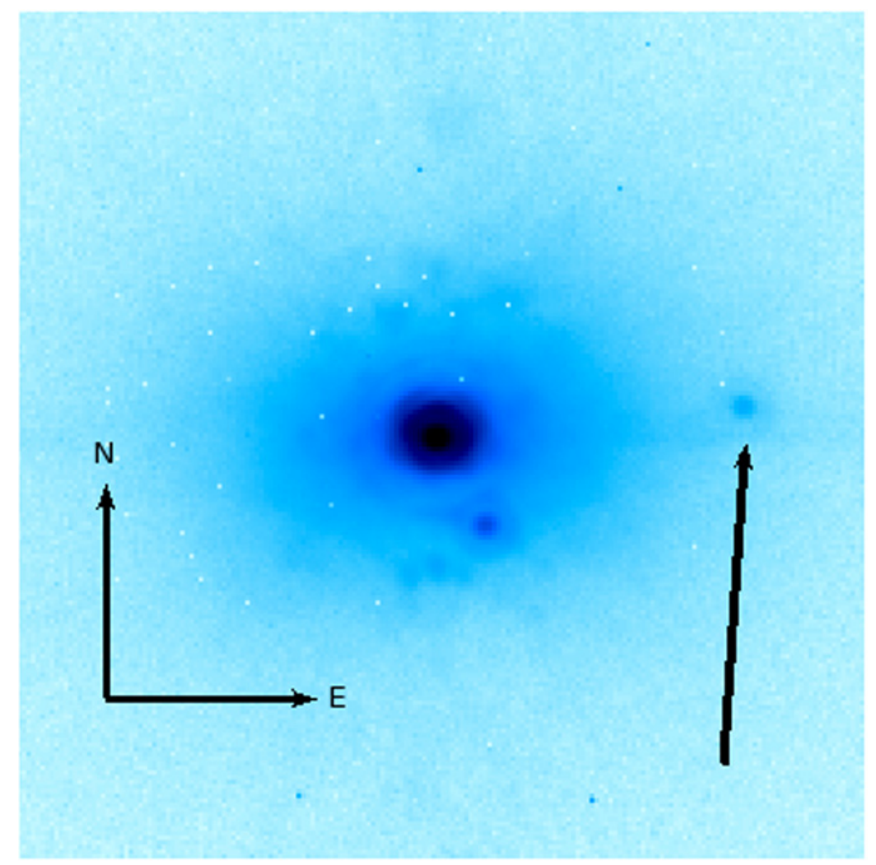

Figure 2. $K s$ images of HD 177830 from the PHARO instrument taken in 2014. An arrow points to the companion. In the image, north is up and east is to the right. The close object to the southeast of the primary is a ghost from a neutral density filter in the camera. This is an approximately $4^{\prime \prime}$ wide subimage from the full $25^{\prime \prime}$ field of view.

\section{SPECTRAL TYPE DETERMINATION OF THE COMPANION}

To create a spectrum of the stellar companion, we performed aperture photometry on the images with sufficient signal-tonoise ratio in the P1640 data cube. We used the same techniques that we have used in our previous papers (e.g., Hinkley et al. 2010; Zimmerman et al. 2010; Roberts et al. 2012). This was done with the aper.pro routine which is part of the IDL astrolib ${ }^{14}$ and is an adaptation of DAOphot (Stetson 1987). A photometry aperture and a sky annulus were centered on the companion. The radius of the photometry aperture was set equal to $3.5 \lambda / D$ rounded to the nearest pixel size, where $\lambda$ is the central wavelength of each image and $D$ is the aperture of the telescope. This corresponds to a radius of 0 ". 18 at the center of $J$ band and 0 ". 24 at the center of $H$ band. The radius of the sky anullus was set to 0 ". 67 ; this is large enough to avoid significant portions of the central point-spread function (PSF) while also avoiding the occultation spot. The width of the sky annulus was set to 10 pixels, or 0 ". 19 . The background was set equal to the average intensity of the pixels in the sky annulus and was subtracted from each pixel in the photometry aperture. The spectrum is the summed power in each slice as function of wavelength. The aper.pro code also produces a measurement error bar based on photon statistics and the error in the background estimation. The extracted spectrum is a convolution of the object spectrum and the spectral response function (SRF). The SRF includes the instrumental response and the absorption due to the Earth's atmosphere. We computed the SRF by using an unocculted observation of HD $177830 \mathrm{~A}$ as a reference source. We extracted the spectrum of HD 177830 A using the aperture photometry method described above. Then we divided the extracted spectrum by a reference spectrum of the same spectral type. HD $177830 \mathrm{~A}$ is a K0IV. We did not have a template for this spectral type, instead we used a K0V spectra from the Infrared Telescope Facility (IRTF) Spectral Library (Rayner et al. 2009). This produces an estimate of the SRF. The companion's measured spectrum is then divided by the SRF and yields the calibrated spectrum of the companion. The portions of the spectrum that overlap with the water bands were removed, as they had large errors.

Since the template spectrum had the incorrect luminosity class, we checked to see how much impact the incorrect luminosity class had on the resulting spectra. We computed the spectrum of HD 177830 B with an SRF from HD 129814 (spectral type G5V), taken two nights later at a similar air mass. The resulting spectrum for HD $177830 \mathrm{~B}$ is the same to within the error bars.

The error bars on the final reduced spectra are the combination of the errors of the measured science spectra and the error on the SRF. The SRF errors come from the combination of the errors of the measured calibration spectra and the errors on the template spectrum. See Roberts et al. (2012) for further discussion of SRFs.

After the spectrum of the companion was extracted from the P1640 data, we compared it against the spectra in the IRTF Spectral Library (Cushing et al. 2005; Rayner et al. 2009). These include FGKM main sequence stars and LT brown dwarfs. The template spectra were binned and smoothed in order to produce the equivalent spectra to having the star observed by P1640. Each spectrum was normalized by its average. Then each template spectrum was compared against the measured spectrum using the sum of the squares of the

\footnotetext{
${ }^{14}$ http://idlastro.gsfc.nasa.gov
} 


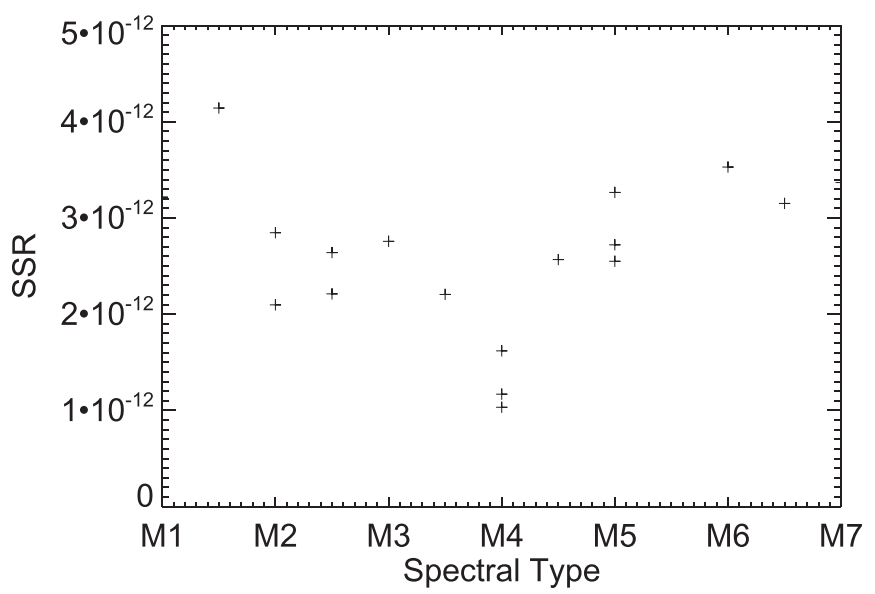

Figure 3. SSR metric from Equation (1) plotted as a function of spectral type for HD 177830. The units of SSR are arbitrary. For some spectral types, the IRTF Spectral Library has multiple stars of that class; this results in multiple data points for several of spectral types. The best fit spectral type is M4V.

residual (SSR) as a metric,

$$
\mathrm{SSR}=\sum_{\lambda} w_{\lambda}\left(S_{\lambda}-R_{\lambda}\right)^{2}
$$

where, $S_{\lambda}$ is the measured spectrum at a given wavelength, $R_{\lambda}$ is the binned reference spectrum at the same wavelength and $w_{\lambda}$ is the weight at the wavelength. The best fit reference spectrum was the one with the minimum value to the metric. The weights were set equal to the inverse of the computed error at each wavelength point. Figure 3 shows the SSR metric plotted as a function of spectral type.

The extracted spectrum of HD $177830 \mathrm{~B}$ is shown in Figure 4. The error bars for each data point are shown in the figure. The error bars are smallest in the $H$-band portion of the spectrum and since the weight of each data point is the inverse of the error bar, this means that the $H$-band data are weighted more heavily than the $J$-band data. This is appropriate for several reasons. The AO performance is improved in the $H$ band and produces a higher quality image. The detector samples the PSF at a higher spatial frequency in $H$-band and finally the coronagraph is optimized for $H$-band observations. This results in a slightly higher contrast in $H$-band (Hinkley et al. 2011) and lower errors.

In Figure 4 we have over plotted the spectra for M2V, M4V, and M6V stars. The best fit (via Equation (1)) for the companion's spectral type is M4V. Several adjacent spectral types have similar fitting metric values and their spectra in Figure 4 appear to fit the data almost as well within the error bars. From this we conclude that the spectral type is M4 $\pm 1 \mathrm{~V}$ with a conservative error bar. This agrees with the earlier photometric determination of M2V-M5V from Eggenberger et al. (2007).

\section{ORBITAL ANALYSIS}

Eggenberger et al. (2007) concluded that the binary star system had common proper motion. With the additional data from Roberts et al. (2012) and this paper, that conclusion is strengthened. Between 2002 and 2014 the companion moved at a rate of $4.8{\text { mas } y r^{-1}}^{-1}$ relative to the primary, while if it was a fixed background star it would have been expected to move

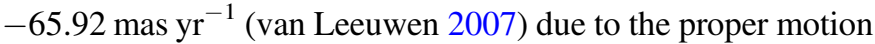
of the primary. This clearly shows that the companion has common proper motion with the primary. We plot the relative motion in Figure 5. From this we can tell the bulk of the motion is to the east, but due to the size of the error bars in relationship with the small amount of motion, we can not tell if the companion has an increasing or decreasing declination. The 2012.4475 data point from P1640 does not line up with the rest of the data. This is probably due to an error in the plate scale for that instrument. The separation corresponds to approximately 90 pixels and a $1 \%$ error in the plate scale would throw the measurement off.

Using the technique of Tokovinin (2014), we can constrain the period of the binary. Using the equation,

$$
P^{*}=\left(\frac{\rho^{3} \pi^{-3}}{M_{1}+M_{2}}\right)^{1 / 2}
$$

where, $P^{*}$ is the probable period based on Kepler's third law, $\rho$ is the measured separation, assumed to be the semimajor axis, $\pi$ is the parallax, and $M_{1}$ and $M_{2}$ are the mass of each star. Tokovinin (2014) carried out simulations with random orbital phases and eccentricities and showed that the median ratio of $\rho / a$ is close to 1 depending slightly on the eccentricity distribution used in the simulations. In all cases, the ratio never exceeds 2 . This results in the ratio of $P^{*} / P_{\text {true }}$ always being less than 3.17. For the case of HD 177830, we use the latest separation measurement from Table 1, and the Hipparcos measured parallax (van Leeuwen 2007). For masses, the spectral type of $\mathrm{M} 4 \mathrm{~V}$ that we derived for the secondary corresponds to a main sequence mass of $0.2 M_{\odot}$ (Reid \& Hawley 2005).

As discussed in the introduction, there are two literature estimates of the mass estimates from spectroscopic modeling: $1.17 \pm 0.10 M_{\odot}$ (Mortier et al. 2013) and $1.37 \pm 0.04 M_{\odot}$ (Jofré et al. 2015). Since the $T_{\text {eff }}$ derived by Mortier et al. (2013) matches the interferometrically derived $T_{\text {eff }}$ of Baines et al. (2008a), we used the mass estimate of Mortier et al. (2013) resulting in a period estimate of 829 years. The mass estimate of Jofré et al. (2015) produces a period of 771 years.

With a semimajor axis of 100-200 AU the stellar companion will not have influenced the formation or dynamical evolution of the exoplanets unless the stellar orbit is extremely eccentric. There is little evidence that the stellar companion has impacted the dynamics of the exoplanets, with semimajor axes of 1.2218 $\pm 0.0008 \mathrm{AU}$ and $0.5137 \pm 0.0003 \mathrm{AU}$. Most of the suggested ways that binary companions interact with exoplanets result in planets with eccentric orbits (Kley \& Nelson 2008) or hot Jupiters (Wu \& Murray 2003; Naoz et al. 2012). In this case, HD $177830 \mathrm{~b}$ has a low eccentricity of $0.009 \pm 0.004$ and appears not to have had its orbit altered by the stellar companion. The inner planet, HD $177830 \mathrm{c}$, has a low to moderate eccentricity of 0.3 , but since it is inside of the orbit of HD $177830 \mathrm{~b}$, it seems unlikely that the stellar companion would have effected its orbit and not that of HD $177830 \mathrm{~b}$. It is possible that the system has additional undetected planets in outer orbits that have been modified by the gravitational pull of HD $177830 \mathrm{~B}$. It is also possible that the system used to have additional outer planets that were ejected from the system due to interactions with the binary (Kaib et al. 2013). Since the eccentricity of HD $177830 \mathrm{~b}$ is almost zero, the system does not appear to be undergoing a Kozai cycle, implying that the 


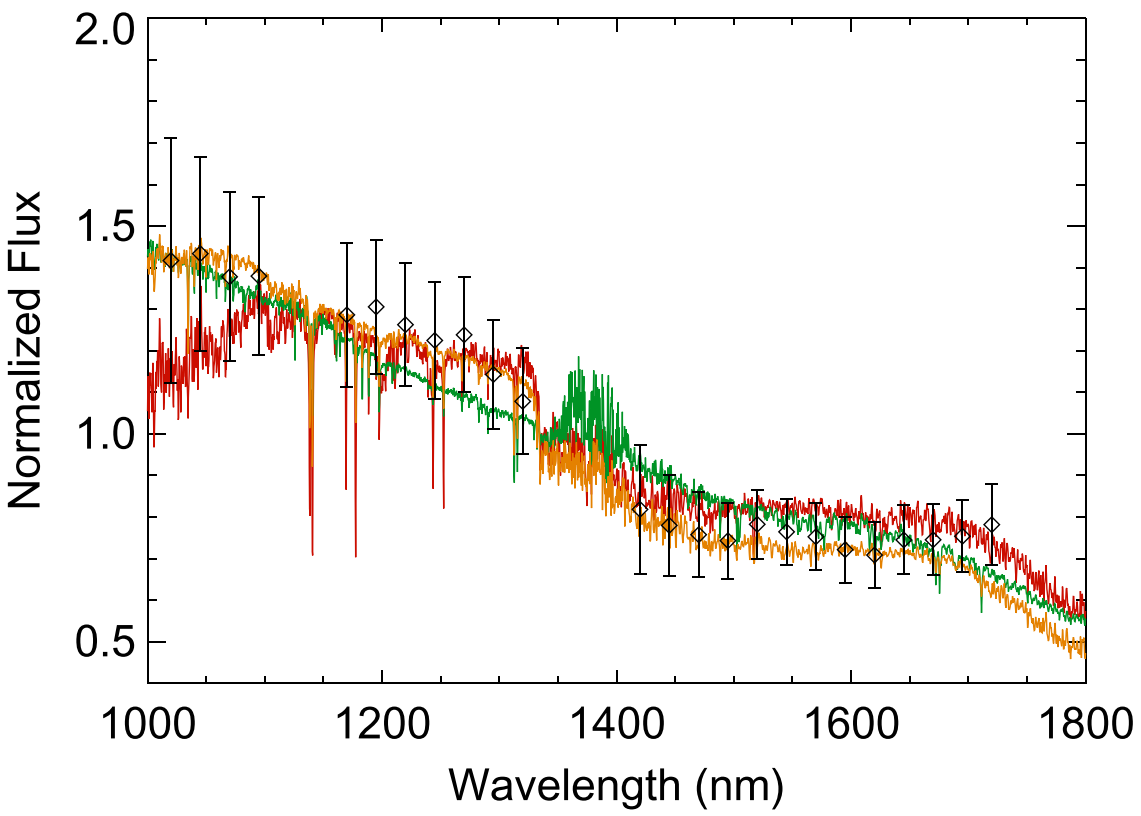

Figure 4. Spectrum extracted from the P1640 data of HD 177830 B. The three over plotted template spectra are Green-M2V, Orange-M4V, and Red-M6V. The determination of the spectral type is $\mathrm{M} 4 \pm 1 \mathrm{~V}$.

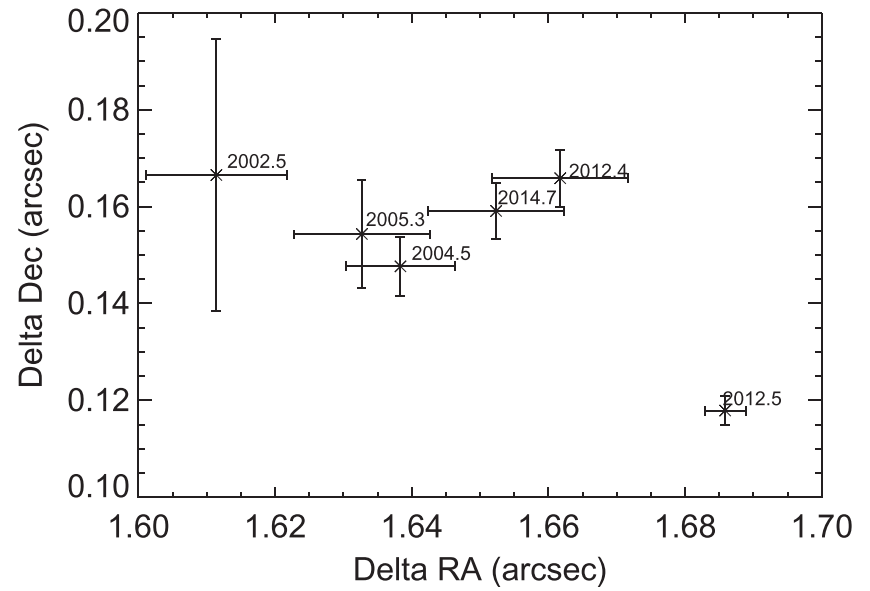

Figure 5. Plot of the relative motion of the companion to the primary star. The primary direction of relative motion is to the east, but we can not tell if the declination is changing or not.

relative inclination of the stellar companion's orbit and that of the planets is less than the critical angle of $39^{\circ} .2$ (Holman et al. 1997).

\section{NULL RESULTS}

In addition to HD 177830, we used P1640 to observe several exoplanet host stars that did not have known companions. These observations happened during the first phase of the P1640 instrument when it was used with PALM-3000 predecessor, PALAO (Dekany et al. 1997; Troy et al. 2000). We did not detect any candidate companions in our 3". 4 field of view. Table 2 lists the name most commonly used in reference to the exoplanet, the Hipparcos number, the Besselian date of the observations and the radius of the field of view in terms of AU. After data reduction with the version of the LOCI speckle suppression software described in (Crepp et al. 2011), all the stars had contrast curves approximately the same as that shown
Table 2

Exoplanet Host Stars with No Detected Companions

\begin{tabular}{lccc}
\hline \hline Name & HIP & Date (UT) & FOV Radius (AU) \\
\hline HD 5319 & 4287 & 2008.8058 & 195 \\
HD 69830 & 40693 & 2009.2054 & 21.2 \\
GJ 849 & 109358 & 2008.5242 & 15.5 \\
\hline
\end{tabular}

in that paper. The contrast is high enough to rule out additional stellar companions within the field of view.

\section{SUMMARY}

We used the P1640 coronagraph and IFU to determine that the secondary in the exoplanet hosting binary system, HD 177830, is a M4 $\pm 1 \mathrm{~V}$ star. We also extracted the astrometry from this measurement as well as two others taken with the PHARO instrument. Combined with published astrometry, we are able to constrain the orbit to a period of approximately 800 years and a semimajor axis of 100-200 AU. This strongly suggests that the binary system has had little to no impact on the known exoplanets in the system. The computed orbit of the system can be improved with additional astrometry, but due to the slow motion of the system, the observations can probably be made every 5-10 years until significant orbital motion is detected.

A portion of the research in this paper was carried out at the Jet Propulsion Laboratory, California Institute of Technology, under a contract with the National Aeronautics and Space Administration (NASA). This work was partially funded through the NASA ROSES Origins of Solar Systems Grant NMO710830/102190. Project 1640 is funded by National Science Foundation grants AST-0520822, AST-0804417, and AST-0908484. The members of the Project 1640 team are also grateful for support from the Cordelia Corporation, Hilary and Ethel Lipsitz, the Vincent Astor Fund, Judy Vale, Andrew Goodwin, and an anonymous donor. C. B. acknowledges support from the Alfred P. Sloan Foundation. We thank the 
staff of the Palomar Observatory for their invaluable assistance in collecting these data. This paper is based on observations obtained at the Hale Telescope, Palomar Observatory. This research made use of the Washington Double Star Catalog maintained at the U.S. Naval Observatory, the SIMBAD database, operated by the CDS in Strasbourg, France and NASA's Astrophysics Data System.

Facilities: Hale (PHARO, Project 1640)

\section{REFERENCES}

Baines, E. K., McAlister, H. A., ten Brummelaar, T. A., et al. 2008a, ApJ, 680, 728

Baines, E. K., McAlister, H. A., ten Brummelaar, T. A., et al. 2008b, ApJ, 682,577

Beichman, C. A., Bryden, G., Rieki, G. H., et al. 2005, ApJ, 622, 1160

Bonavita, M., \& Desidera, S. 2007, A\&A, 468, 721

Bryden, G., Beichman, C. A., Carpenter, J. M., et al. 2009, ApJ, 705, 1226

Butler, R. P., Wright, J. T., Marcy, G. W., et al. 2006, ApJ, 646, 505

Crepp, J. R., Puyeo, L., Brenner, D., et al. 2011, ApJ, 729, 132

Cushing, M. C., Rayner, J. T., \& Vacca, W. D. 2005, ApJ, 623, 1115

Dekany, R., Roberts, J., Burruss, R., et al. 2013, ApJ, 776, 130

Dekany, R., Wallace, K., Brack, G., Oppenheimer, B. R., \& Palmer, D. 1997, Proc. SPIE, 3126, 269

de Silva, L., Girardi, L., Pasquini, L., et al. 2006, A\&A, 458, 609

Dodson-Robinson, S. E., Beichman, C. A., Carpenter, J. M., \& Bryden, G. 2011, AJ, 141, 11

Eggenberger, P., Udry, S., Chauvin, G., et al. 2007, A\&A, 474, 273

Ghezzi, L., Cunha, K., Schuler, S. C., \& Smith, V. V. 2010, ApJ, 725, 721

Hayward, T. L., Brandl, B., Pirger, B., et al. 2001, PASP, 113, 105

Hinkley, S., Oppenheimer, B. R., Brenner, D., et al. 2010, ApJ, 712, 421

Hinkley, S., Oppenheimer, B. R., Zimmerman, N., et al. 2011, PASP, 123, 74

Holman, M., Touma, J., \& Tremaine, S. 1997, Natur, 386, 254

Jofré, E., Petrucci, R., Saffe, C., et al. 2015, A\&A, 574, A50

Kaib, N. A., Raymon, S. N., \& Duncan, M. 2013, Natur, 493, 381

Kley, W., \& Nelson, R. P. 2008, A\&A, 486, 617
Lu, P. K., Demarque, P., van Altena, W., McAlister, H., \& Hartkopf, W. 1987, AJ, 94, 1318

Meschiari, S., Laughlin, G., Vogt, S. S., et al. 2011, ApJ, 727, 117

Mortier, A., Santos, N. C., Sousa, S. G., et al. 2013, A\&A, 557, A70

Naoz, S., Farr, W. M., \& Rasio, F. A. 2012, ApJL, 754, L36

Raghavan, D., Henry, T. J., Mason, B. D., et al. 2006, ApJ, 646, 523

Rayner, J. T., Cushing, M. C., \& Vacca, W. D. 2009, ApJS, 185, 289

Reffert, S., \& Quirrenbach, A. 2011, A\&A, 527, A140

Reid, I. N., \& Hawley, S. L. 2005, New Light on Dark Stars (2nd ed.; Berlin: Springer)

Roberts, L. C., Jr., Rice, E. L., Beichman, C. A., et al. 2012, AJ, 144, 14

Roberts, L. C., Jr., Tokovinin, A., Mason, B. D., Hartkopf, W. I., \& Riddle, R. L. 2015, AJ, submitted

Roberts, L. C., Jr., Turner, N. H., Bradford, L. W., et al. 2005, AJ, 130, 2262

Roberts, L. C., Jr., Turner, N. H., ten Brummelaar, T. A., Mason, B. D., \& Hartkopf, W. I. 2011, AJ, 142, 175

Saffe, C., Gómez, M., \& Chavero, C. 2005, A\&A, 443, 609

Sivaramakrishnan, A., \& Oppenheimer, B. R. 2006, ApJ, 647, 620

Stetson, P. B. 1987, PASP, 99, 191

Takeda, G., Ford, E. B., Sills, A., et al. 2007, ApJS, 168, 297

Tanner, A., Beichman, C., Bryden, G., Lisse, C., \& Lawler, S. 2009, ApJ, 704, 109

ten Brummelaar, T. A., Mason, B. D., Bagnuolo, W. G., Jr., et al. 1996, AJ, 112,1180

ten Brummelaar, T. A., Mason McAlister, H. A., Roberts, L. C., Jr., et al. 2000, AJ, 119, 2403

Tokovinin, A. 2014, AJ, 147, 86

Trilling, D., Bryden, G., Beichman, C. A., et al. 2008, ApJ, 674, 1086

Troy, M., Dekany, R. G., Brack, G., et al. 2000, Proc. SPIE, 4007, 31

Valenti, J. A., \& Fischer, D. A. 2005, ApJS, 159, 141

van Leeuwen, F. 2007, A\&A, 474, 653

Vogt, S. S., Marcy, G. W., Butler, R. P., \& Apps, K. 2000, ApJ, 536, 902

Wright, J. T., Marcy, G. W., Fischer, D. A., et al. 2007, ApJ, 657, 533

Wu, Y., \& Murray, N. 2003, ApJ, 589, 605

Zimmerman, N., Brenner, D., Oppenheimer, B. R., et al. 2011, PASP, 123, 746

Zimmerman, N., Oppenheimer, B. R., Hinkley, S., et al. 2010, ApJ, 709,733

Zucker, S., \& Mazeh, T. 2002, ApJL, 568, L113 